\title{
Constructing Confidence Intervals For Flexible Budget Cost Estimates
}

Mitchell H. Raiborn, (E-mail: mhr@bradley.edu), Bradley University Robert C. Scott, (E-mail: rcs@bradley.edu), Bradley University

G. R. Cluskey Jr., (E-mail: bobcluskey@grccpa.com), Troy University, Dothan

\begin{abstract}
A multiple-cost flexible budget can be constructed using either the Aggregate Cost Analysis Method or the Component Flexible Budget Method. This paper derives and illustrates the use of a confidence interval formula for an annual cost estimate that is developed by summing 12 monthly flexible budget estimates.
\end{abstract}

\section{INTRODUCTION}

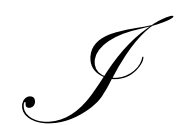

$\mathrm{n}$ a previous journal article, the authors (Cluskey, et. al. 2000, 35-47) demonstrated two different methods of constructing multiple-cost flexible budgets using PC-based regression analysis. Simple linear regression analysis can be used to construct multiple-cost flexible budgets for an aggregate cost that consists of several component cost elements. A multiple-cost flexible budget can be constructed using either the Aggregate Cost Analysis Method or the Component Flexible Budget Method. This paper derives and illustrates the use of a confidence interval formula for an annual cost estimate that is developed by summing 12 monthly flexible budget estimates.

The statistical formula for constructing a confidence interval for a monthly cost estimate is well-known. Using this expression, we have created a confidence interval formula for an annual cost estimate that is constructed by summing 12 monthly flexible budget estimates. This confidence interval formula is an original contribution to the body of knowledge in statistical cost estimation. A numerical example is presented to demonstrate the practical value of the annual cost confidence interval formula. This formula has practical applications in both management accounting and auditing practice.

\section{CONSTRUCTING MULTIPLE-COST FLEXIBLE BUDGETS}

A multiple-cost flexible budget estimates the combined amount of several component costs to be incurred at different levels of activity during a specified time period. From the standpoint of cost analysis procedures, multiple-cost flexible budgets can be developed in one of two ways:

1. Aggregate Cost Analysis Method. Disregard the cost behavior and flexible budgets that may have been defined for component cost factors and develop an autonomous flexible budget equation by analyzing aggregate cost amounts for previous time periods.

2. Component Flexible Budget Method. Formulate flexible budget equations for each individual cost factor and combine the resulting flexible budget equations, if the same independent variable (cost driver) is used for all component costs. Otherwise, cost estimates from component cost flexible budgets are added to measure the aggregate cost factor at some specific level of activity.

Flexible budget point estimates for an aggregate cost factor will be equal under both methods only if the same cost driver (independent variable) is used in all component cost flexible budget equations. It is unlikely that a single cost driver could provide reasonably accurate flexible budgets for all component costs comprising an aggregate cost factor. Thus, as a practical matter, multiple-cost flexible budgets developed under each method will usually provide different aggregate cost estimates. If flexible budgets for individual cost factors are not needed for 
cost control reports, then it is more expedient to develop a multiple-cost flexible budget using the aggregate cost analysis method. If a confidence interval is needed for the aggregate cost estimate, then regression analysis must be applied to aggregate cost data to determine the standard error of the estimate. The standard error of estimate based on aggregate cost analysis will be less than the sum of standard errors from the component cost flexible budgets.

Information requirements and data-management capabilities within a company will influence the method used to develop multiple-cost flexible budgets. The guideline that costs should be related to their most causal factor is very important for flexible budget equations to be used in cost control analysis. For use in cost control reports, flexible budgets should be developed for each cost factor in every responsibility unit within a company, using the independent variables giving the best $\mathrm{r}^{2}$ and lowest standard error of estimate. The component flexible budget method must be used to estimate aggregate costs in these control reports. It is a practical necessity in control reports that an aggregate cost must equal the sum of its component costs. Cost control reports are ex-post in character, since actual costs for a past period are compared with flexible budget measures of what the costs should have been at the actual volume level. Multiple-cost flexible budgets used for planning purposes are ex-ante in character and thus differ from the component cost flexible budgets later used to prepare detailed cost control reports.

The aggregate cost analysis method can be applied to develop multiple-cost flexible budgets that will be used exclusively for planning and future cost prediction purposes. Flexible budgets derived from aggregate cost analysis greatly simplify the data management problem involved in combining the flexible budgets of component cost factors. A company with 12 manufacturing plants having 30 departments in each plant with 50 individual cost factors in each department requires 18,000 cost behavior equations to define the flexible budgets of all individual cost items ( 12 X $30 \times 50)$. Aggregate overhead costs classified by department can be represented by 30 flexible budget equations at each plant. By using the aggregate cost analysis method, the 30 equations at each plant can be based on the one independent variable that is most appropriate for each department.

When a sufficient number of component costs are aggregated, many complex forms of cost behavior are merged to yield a multiple-cost measure that can be accurately represented by a single flexible budget equation using one independent variable. Thus, the aggregate cost analysis method is most useful in defining multiple-cost flexible budgets for planning purposes in which the objective is to predict aggregate costs as accurately and efficiently as possible. The use of different flexible budgets for planning purposes and for cost control reporting systems is practical, effective, and conceptually justified (Cluskey, et al 2000, 35-47).

\section{CONSTRUCTING CONFIDENCE INTERVALS FOR ANNUAL COST ESTIMATES BASED ON MONTHLY FLEXIBLE BUDGET EQUATIONS}

If data for a cost factor for twelve monthly time periods are available, then a point estimate of annual costs may be easily generated as follows:

$$
\hat{y}_{j, A n}=n a_{j}+b_{j} \sum_{i=1}^{n} x_{i}
$$

$$
\begin{array}{cc}
\mathrm{i}=1,2, \ldots, \mathrm{n} ; & \mathrm{j}=1,2, \ldots, \mathrm{m} \\
\text { (time periods) } & \text { (cost factors) }
\end{array}
$$

where $\hat{y}_{j, A n}$ is the predicted annual cost for the jth cost factor, $\mathrm{a}_{\mathrm{j}}$ is the estimated monthly "fixed cost" component for the jth cost factor, and $b_{j}$ is the estimate of the "incremental cost" component for the jth cost factor. Since $3 \mathrm{x}_{\mathrm{i}}$ stands for the summation of, in this case, twelve monthly values of the independent variable (cost driver) in the above equation, it may be rewritten as

$$
\hat{y}_{j, A n}=n a_{j}+n b_{j} \bar{x}
$$

We will now demonstrate that the computational formula for the confidence interval for an annual cost estimate is unexpectedly simplified, but very different from the corresponding expression for a confidence interval for an estimated cost of a single month. 
The expression that gives a confidence interval for a monthly cost estimate is

$$
y_{j i}=\hat{y}_{j i} \pm\left(t_{\alpha / 2}^{n-2}\right) S_{e} \sqrt{\frac{1}{n}+\frac{\left(x_{i}-\bar{x}\right)^{2}}{\sum x_{i}-n \bar{x}^{2}}+1}
$$

where $S_{\mathrm{e}}$ is the standard error of the estimate for $\hat{y}_{j i}$.

In this formula, the expression to the right of the value of the t-statistic is the sample estimate of the population standard deviation of dependent variable values (y) for some given value of the independent variable (x), also known as the standard error of the forecast. In most cases, the numerical value of the expression under the square root radical approximates 1.0 and is often disregarded in practice. Squaring this expression yields a sample estimate of the population variance

$$
\left(\sigma_{y_{j i}}^{2} \mid x_{i}\right) \text { given some value of } \mathrm{x} \text { : }
$$

$$
\text { Estimated } \sigma_{y_{j i} \mid x_{i}}^{2}=S_{e}^{2}\left[\frac{1}{n}+\frac{\left(x_{i}-\bar{x}\right)^{2}}{\sum x_{i}-n \bar{x}^{2}}+1\right]
$$

where $S_{e}^{2}$ is the sample variance of estimate.

It is well known that if two variables have a zero covariance, then the variance of the sum of the variables equals the sum of the variances of the variables. This may be written as

$$
\sigma_{A+B}^{2}=\sigma_{A}^{2}+\sigma_{B}^{2} \quad \text { if } \quad \sigma_{A, B}=0 .
$$

Since a predicted annual cost equals the sum of 12 predicted monthly costs, we may generalize (4) above on the basis of (5) above as follows:

$$
\sigma_{y_{j, A n}}^{2}=\sum \sigma_{y_{j i} \mid x_{i}}^{2}
$$

Since $\hat{\sigma}_{y_{j, A n}}^{2}$ is the sample estimate of (6) above, an expression analogous to (4) above is

$$
\hat{\sigma}_{y_{j, A n}}=\sum S_{e}^{2}\left[\frac{1}{n}+\frac{\left(x_{i}-\bar{x}\right)^{2}}{\sum x_{i}^{2}-n \bar{x}^{2}}+1\right]
$$

By the distributive law of multiplication,

$$
\hat{\sigma}_{y_{j, A n}}^{2}=\sum S_{e}^{2}\left(\frac{1}{n}\right)+\sum S_{e}^{2} \frac{\left(x_{i}-\bar{x}\right)^{2}}{\sum x_{i}^{2}-n \bar{x}^{2}}+\sum S_{e}^{2}(1)
$$

Since $\sum\left(\frac{1}{n}\right)=1$, and since $S_{e}^{2}$ is constant, then

$$
\sum S_{e}^{2}\left(\frac{1}{n}\right)=S_{e}^{2}(1)
$$

The expression $\sum x^{2}-n \bar{x}^{2}$ is a simple computational formula for "sum of squared deviations" of values of $\mathrm{x}$ from their mean ( $\square$ ). This can also be written as $\square$ (

Thus, $\sum\left(x_{i}-\bar{x}\right)^{2}=\sum x_{i}^{2}-n \bar{x}^{2}$. Therefore from (8) above, and since $S_{e}^{2}$ is constant 


$$
\sum\left(S_{e}^{2}\right) \frac{\left(x_{i}-\bar{x}\right)^{2}}{\sum x_{i}^{2}-n \bar{x}^{2}}=S_{e}^{2} \sum \frac{\left(x_{i}-\bar{x}\right)^{2}}{\sum x_{i}^{2}-n \bar{x}^{2}}
$$

But since $\sum\left(x_{i}-\bar{x}\right)^{2}=\sum x_{i}^{2}-n \bar{x}^{2}$, and given that $\sum x_{i}{ }^{2}-n \bar{x}^{2}$ is

constant, then

$$
\sum \frac{\left(x_{i}-\bar{x}\right)^{2}}{\sum x_{i}^{2}-n \bar{x}^{2}}=\frac{\sum\left(x_{i}-\bar{x}\right)^{2}}{\sum x_{i}^{2}-n \bar{x}^{2}}=\frac{\sum x_{i}^{2}-n \bar{x}^{2}}{\sum x_{i}^{2}-n \bar{x}^{2}}=1 .
$$

Thus (10) above may be simplified as follows

$$
S_{e}^{2} \sum \frac{\left(x_{i}-\bar{x}\right)^{2}}{\sum x_{i}^{2}-n \bar{x}^{2}}=S_{e}^{2}(1)
$$

Since $S_{e}^{2}$ is a constant, in (8) above $\sum\left(S_{e}^{2}\right)(1)$ may be written as

$$
\sum S_{e}^{2}(1)=S_{e}^{2} \sum(1)
$$

Since $\sum(1)=n$ then (13) may be written as

$$
\sum S_{e}^{2}(1)=S_{e}^{2}(n)
$$

On the basis of (9), (12), and (14) above, (8) above can be written as

$$
\hat{\sigma}_{y_{j, A n}}^{2}=S_{e}^{2}(1)+S_{e}^{2}(1)+S_{e}^{2}(n) .
$$

By the distributive law of multiplication,

$$
\hat{\sigma}_{y_{j, A n}}=S_{e}^{2}(n+2)
$$

An "annual cost" analogue of (3) above is therefore

$$
y_{j, A n}=\hat{y}_{j, A n} \pm\left(t_{\propto \propto / 2}^{n-2}\right) S_{e} \sqrt{n+2}
$$

The confidence interval for annual cost may be formally written

$$
\hat{y}_{j . A n}-\left(t_{\alpha / 2}^{n-2}\right) S_{e} \sqrt{n+2} \leq y_{j, A n} \leq \hat{y}_{j . A n}+\left(t_{o d 2}^{n-2}\right) S_{e} \sqrt{n+2} .
$$

The probability that actual annual cost will fall within this range of values is 1- $\alpha$.

\section{A PRACTICAL APPLICATION DEMONSTRATED}

The setting and given facts:

A. Monthly profit planning budgets (12) for a semivariable cost. This cost could be a single cost component or it could represent the sum of multiple cost components.

B. The cost driver selected is production volume in units.

C. Flexible budget formula: $\mathrm{Y}=\$ 847$ per month $+\$ .861$ per unit. This monthly flexible budget equation is based on operating data for the preceding 12 months. This cost corresponds with Cost Factor F [Cluskey, et al 2000, 36,38].

D. Standard Error of Estimate for $\mathrm{Y}=\$ 3,324 ; \mathrm{R}^{2}=.8357$ 
Table 1

Monthly Cost Budget Data

\begin{tabular}{|c|c|c|c|c|}
\hline Monthly Budgets & Production Volume & Fixed Cost & Variable Cost & Total Cost \\
\hline January & 18,200 & $\$ 847$ & $\$ 15,670$ & $\$ 16,517$ \\
\hline February & 19,000 & 847 & 16,359 & 17,206 \\
\hline March & 23,700 & 847 & 20,406 & 21,253 \\
\hline April & 26,400 & 847 & 22,730 & 23,577 \\
\hline May & 30,100 & 847 & 25,916 & 26,763 \\
\hline June & 32,900 & 847 & 28,327 & 29,174 \\
\hline July & 4,500 & 847 & 3,875 & 4,722 \\
\hline August & 8,200 & 847 & 7,060 & 7,907 \\
\hline September & 10,000 & 847 & 8,610 & 9,457 \\
\hline October & 13,700 & 847 & 11,796 & 12,643 \\
\hline November & 16,100 & 847 & 13,862 & 14,709 \\
\hline December & 16,900 & 847 & 14,551 & 15,398 \\
\hline Totals & $\mathbf{2 1 9 , 7 0 0}$ & $\mathbf{\$ 1 0 , 1 6 4}$ & $\mathbf{\$ 1 8 9 , 1 6 2}$ & $\mathbf{\$ 1 9 9 , 3 2 6}$ \\
\hline
\end{tabular}

The point estimate of total annual cost for this cost factor is $\$ 199,326$, which is the sum of the 12 monthly cost budgets. A 95\% confidence interval for this annual cost estimate is constructed as follows using the standard error of the estimate $(\$ 3,324)$ and the appropriate $t$-value for a two-tailed distribution. (See equation 17 above).

The $t$-value for $\alpha=.05$ and 10 degrees of freedom is $2.228 ; S_{\mathrm{e}}$ is $\$ 3,342$; and $\sqrt{12+2}=3.741657$.

The LCL is $\$ 199,326-(2.228)(\$ 3,342)(3.741657)=\$ 171,466$.

The UCL is $\$ 199,326+(2.228)(\$ 3,342)(3.741657)=\$ 227,186$.

We are $95 \%$ confident that actual total cost for the year will fall between the LCL of \$171,466 and the UCL of $\$ 227,186$.

\section{USES OF ANNUAL COST CONFIDENCE INTERVALS}

From a profit planning standpoint, there are several possible uses of the LCL (lower confidence limit), the point estimate of total annual cost, and the UCL (upper confidence limit). The "most optimistic" (best-case) profit plan could incorporate the LCL of the annual cost item. The "most likely" profit plan or budget for a one year period should use the point estimate of total annual cost, $\hat{y}_{a n}$. The "worst case" profit plan could incorporate the UCL of the estimated annual cost measure. This range of best-case, most-likely, and worst-case creates flexibility in the evaluation of annual budget results in the same manner that three-measures of alternatives can be evaluated in a decision-making situation. The monthly flexible budget equations are also very useful in continuous or rolling budgets having a continuous annual time frame. The confidence level expression in (18) indicates how to measure the LCL and UCL for an annual cost estimate that is developed by summing 12 monthly flexible budget estimates.

As a test of reasonableness with analytical procedures, independent auditors can use the expression in (18) to measure the LCL and UCL of a specific annual cost item, such as repairs and maintenance expense. The reasonableness of the recorded actual annual cost is based on whether that amount lies within the bounds of the LCL and UCL measured using monthly flexible budget equations and actual values of the cost driver (independent variable). Additionally, independent auditors could apply the confidence interval expression in (18) to expense account balances as part of their financial analysis during initial testing of a client's unaudited financial statements and industry data to assess the risk of misstatements. 
Because of the $\sqrt{n+2}$ element in (17), we believe that the formula in (17) makes an original contribution to the body of knowledge in statistical cost estimation. Both ex-ante profit planning estimates and expost audit conclusions could be materially erroneous if the confidence interval for an annual cost estimate is not measured correctly. A confidence interval developed without the $\sqrt{n+2}$ factor would be too narrow in range and would overstate the LCL and understate the UCL. These misstatements would cause incorrect estimates to be used in the "best case" and "worst case" profit plans that many firms evaluate.

An incorrect LCL and UCL could cause a Type II decision error in auditing such that an actual annual expense that is truly reasonable in amount would be judged unreasonable when the total expense fails to lie within the bounds of the incorrectly measured confidence interval. The Type II decision error in auditing would lead to unnecessary substantive testing of the expense item, thus wasting time and money.

\section{CONCLUSIONS}

A multiple-cost flexible budget estimates the combined amount of several component costs to be incurred at different levels of activity during a specified time period. A multiple-cost flexible budget using simple linear regression analysis can be developed by using the Aggregate Cost Analysis Method or the Component Flexible Budget Method (Cluskey, et. al. 2000, 46). By applying the mathematics of regression analysis to the component flexible budget method, it is known that the standard error of estimate of the aggregate cost will not equal the sum of the standard errors of the individual cost elements that comprise the aggregate cost. The standard error of the estimate must be used when constructing confidence intervals for a cost estimate.

Confidence intervals are useful measures for cost estimates in both budgeting and auditing practice. The statistical formula for constructing a confidence interval for a single monthly cost estimate is well-known. Based on this expression, we have created a formula to construct a confidence interval for an annual cost estimate that is developed by summing 12 monthly flexible budget estimates. This formula is presented in equation (17). Because of the $\sqrt{n+2}$ element, the annual cost confidence interval formula in equation (17) is an original contribution to the body of knowledge in statistical cost estimation. A numerical application of this expression was demonstrated using a monthly flexible budget equation for a semi-variable cost having parameters measured using simple linear regression. The confidence interval formula in equation (17) has practical applications in management accounting and auditing practice. Incorrectly measured confidence intervals for an annual cost estimate produce unreliable budgets and could cause a Type II decision error in auditing.

\section{REFERENCES}

1. Cluskey Jr., G. R., Raiborn, M. H., \& Modianos, D. T. 2000. Multiple-Cost Flexible Budgets and PC-Based Regression Analysis. Journal of Cost Management. 14(4): 35-47.

2. Guercio, J. P. \& Jordan, J. M. 1992. Cost Forecasting with Regression Analysis. CFO. (February): 51-54.

3. Hirsch, Jr., M. L. 1993. Advanced Management Accounting. 2ed. South-Western: Ohio. Chapters 4 \& 5:156-232.

4. Jensen, R. E. 1967. A Multiple Regression Model for Cost Control: Assumptions and Limitations. Accounting Review. 42(2): 265-274.

5. Jones, L. F. 1988. Competitor Cost Analysis at Caterpillar. Management Accounting. (October): 32-38.

6. Jones, L. F. 1991. Product Costing at Caterpillar. Management Accounting. (February): 34-40.

7. Kaplan, R. S. \& Atkinson, A. A. 1989. Advanced Management Accounting. 2ed, Prentice Hall, New Jersey. Chapters 4 \& 5: 93-152.

8. Merchant, K. A. \& Shields, M. D. 1993. When and Why to Measure Costs Less Accurately to Improve Decision Making. Accounting Horizons. 7(2): 76-81.

9. Novin, A. M. 1992. Applying Overhead: How to Find the Right Bases and Rates. Management Accounting. 73(9): 40-43. 